\title{
Differential Sensitivity of Endothelial Cells of Various Species to Apoptosis Induced by Gene Transfer of Fas Ligand: Role of Flip Levels
}

\author{
Delphine Bouchet, ${ }^{1}$ Laurent Tesson, ${ }^{1}$ Séverine Ménoret, ${ }^{1}$ Béatrice Charreau, ${ }^{1}$ Patrick Mathieu, ${ }^{1}$ \\ Hideo Yagita, ${ }^{2}$ Ghislaine Duisit, ${ }^{3}$ and Ignacio Anegon ${ }^{1}$ \\ ${ }^{1}$ Institut National de la Santé Et de la Recherche Médicale U437 and Institut de Transplantation et de \\ Recherche en Transplantation, Nantes, France \\ ${ }^{2}$ Juntendo University School of Medicine, Tokyo, Japan \\ ${ }^{3}$ Laboratoire de Thérapie Génique Nantes, France \\ Accepted August 13, 2002
}

\begin{abstract}
Background: Fas ligand expression by cells of the vessel wall has been proposed to play a role in normal and pathologic conditions. Genetic engineering of vascularized organs for endothelial cell (EC) expression of FasL could protect the endothelium and underlying tissues from infiltrating Fas + leukocytes. Nevertheless, the endogenous expression of FasL by ECs of different species and the potential deleterious effects of enforced FasL expression by ECs are largely unknown. In human ECs, levels of FLICE/caspase 8-inhibitory protein (FLIP) have been shown to control apoptosis mediated by Fas.

Materials and Methods: Cell surface expression of FasL in rat, mouse, human, and pig ECs was obtained using recombinant adenoviruses or transient plasmid transfection assays. FasL expression was evaluated by FACS analysis and cytotoxicity assays. Apoptosis was evaluated using annexin V, TUNEL, and cytotoxicity assays. FLIP levels
\end{abstract}

were evaluated by Western blot analysis and overexpression was obtained by transient transfection.

Results: Analysis of ECs from different species showed that FasL was predominantly present in the cytoplasm, and depending on the species, little or no cell surface expression was detected. Enforced cell surface expression of FasL on rat or mouse ECs, either in culture or within the vessel wall resulted in massive apoptosis. In contrast, porcine or human ECs were completely resistant to apoptosis mediated by Fas-FasL interaction. Markedly reduced FLIP levels were observed in rat and mouse ECs compared to human and porcine ECs. Overexpression of FLIP in rat ECs conferred protection against cell surface expression of FasL. Conclusions: The consequences of FasL overexpression depend on the subcellular compartment and species in which FasL enforced expression is targeted and this is at least partially related to FLIP levels.

\section{Introduction}

Fas ligand (FasL, APO-1L, or CD95L) is a type II transmembrane protein of 37-40 kDa that belongs to the tumor necrosis factor (TNF) family (1). FasL interacts with its receptor Fas (APO-1, CD95), a member of the TNF receptor family expressed in many different cell types. Ligation of Fas induces apoptosis through recruitment of the adaptor protein Fasassociated death domain (FADD) and caspase- 8 (or FLICE), which activates downstream caspases that result in irreversible cell damage (1). FasL plays a major role in cytotoxicity mediated by cytotoxic $\mathrm{T}$ lymphocytes and natural killer (NK) cells and maintenance of immune homeostasis (1). FasL was originally reported to be expressed on the cell surface of activated T cells and resting NK cells (1), but more recent studies have shown a more widespread expression on other cell types, such as Sertoli cells,

D. B. and L. T. contributed equally to this work.

Address correspondence to Ignacio Anegon, INSERM U437, 30 boulevard Jean Monnet, 44093 Nantes Cedex 01, France. Phone: (33) 2-40-08-74-15; fax: (33) 2-40-08-74-11;

e-mail: ianegon@nantes.inserm.fr. cells from the eye, placenta cells, and astrocytes (2). FasL expression in nonimmune tissues has been shown to participate in organ-specific immune privilege by preventing infiltration of these tissues by Fas + leukocytes (3). This property of FasL has been exploited with success by ectopically expressing FasL to prevent tissue destruction in autoimmune diseases (4) or in some transplantation models (5-8). Nevertheless, de novo expression of FasL by tumor cells (9) or by certain grafts from FasL transgenic animals $(10,11)$ has also been associated with inflammation and accelerated rejection. FasL has also recently been described as being expressed constitutively in the cytoplasm of hematopoietic cells, such as monocytes (12), and NK cells (13).

Endothelial cell (EC) apoptosis has been shown to be an important feature in several vascular and immune-mediated pathologic conditions, such as endotoxic shock (14), transplant coronary disease (15), thrombotic thrombocytopenic purpura (16), IL-2induced vascular leak syndrome (17), atherosclerosis (18), and acute graft versus host disease (GVHD) (19).

Cultured primary human ECs from aortas or umbilical vein origin have recently been described 
as constitutively expressing cell-surface FasL (20), whereas murine liver ECs do not show expression of FasL (21). TNF- $\alpha$ was shown to down-regulate cellsurface expression of FasL in human ECs, promoting tissue infiltration by leukocytes (20). Human ECs have been described as resistant to Fas-mediated apoptosis in vitro $(20,22)$ and following culture with oxidized-low-density lipoproteins (23) or extracellular matrix detachment (24), become susceptible to Fas-dependent apoptosis. In contrast, resting human subretinal choroidal capillary ECs were sensitive to apoptosis (25). Murine ECs in resting conditions are sensitive to apoptosis induced by antiFas monoclonal antibodies (mAbs) in vitro (21) and importantly in vivo (19). Sensitivity of human EC $(24,26,27)$ to Fas-mediated apoptosis has been shown to be dependent on down-regulation of FLICE/caspase 8-inhibitory protein (FLIP), an endogenous dominant-negative inhibitor of caspase-8. Fas and FasL have been shown to be directly responsible for EC apoptosis in some immunemediated pathologic conditions $(15,17,19)$.

On the one hand, these results suggest that continuous expression of FasL by ECs could protect underlying tissues during inflammation by preventing leukocyte extravasation. Therefore, continuous expression of cell-surface FasL by ECs, either through generation of transgenic animals or after gene transfer, may provide protection against tissue destruction during inflammation by inducing apoptosis of Fas + infiltrating leukocytes in situations such as allograft rejection, sepsis, and atherosclerosis. The generation of transgenic animals expressing FasL by ECs has been proposed as a therapeutic strategy for xenotransplantation (28). On the other hand, expression of FasL by ECs could be deleterious because ECs can, under certain circumstances, be susceptible to apoptosis mediated by autocrine Fas-FasL interactions $(23,26)$.

The aims of this work were to analyze the expression of FasL by rat heart microvascular and aorta ECs, to genetically engineer expression of FasL at high levels at the cell surface of rat ECs using transgenic technology and adenoviral vectors and finally, to analyze the consequences of FasL expression on ECs. The rat was chosen as a model because of its convenient size for transplantation of vascularized organs and for the availability of transgenic technology $(29,30)$. Results obtained with rat ECs were compared to those of ECs of mouse, human, and pig origin.

Our results show that resting cultured ECs of rat, mouse, human, and pig origin express FasL mostly intracellularly and that high level cellsurface expression of FasL in rat or mouse but not in human or pig ECs results in apoptosis. FLIP levels in rat and mouse ECs were greatly diminished compared to human and porcine ECs and transfection of rat ECs with FLIP partially protected the cells from Fas-mediated apoptosis.
These results show that enforced expression of FasL in ECs can result in apoptosis depending on the species origin of the ECs and underline the fact that Fas-mediated apoptosis of ECs represent an important pathologic mechanism of immune disorders.

\section{Materials and Methods Cell Culture}

Primary ECs from rat hearts and aortas were obtained and cultured as previously described in detail (30). Porcine aortic ECs and human umbilical vein EC (HUVEC) were obtained and cultured by the same procedure described for rat aortic ECs. Primate COS cells, murine A20 cells (31), human Jurkat, murine L5178Y, and L5178Y-FasL cells (expressing or not cell-surface rat or mouse FasL) (32), SVHUK (SV40 immortalized human vascular smooth muscle cells positive for $\alpha$-actin and negative for EC markers) were cultured in RPMI culture medium containing $10 \%$ heat-inactivated FCS and $1 \mathrm{mM}$ of glutamine at $37{ }^{\circ} \mathrm{C}, 5 \% \mathrm{CO}_{2}$. Jurkat cells were stimulated with concanavalin A for $48 \mathrm{hr}$. The mouse 2F2B endothelial cell line (33) (American Tissue Culture Collection, Bethesda, MD, USA) was kindly provided by Dr. M. Soares (Institut Gulbenkian de Cienca, Portugal) and was grown in DMEM culture medium containing $10 \%$ heat-inactivated FCS and $1 \mathrm{mM}$ of glutamine at $37{ }^{\circ} \mathrm{C}, 5 \% \mathrm{CO}_{2}$.

\section{Antibodies}

Antibodies used in this study were mouse mAbs against human FasL cross-reacting with mouse and rat FasL, clone 33 (IgGl, Transduction Laboratories, Lexington, KY, USA) and clone G247-4 (IgGl, PharMingen, San Diego, CA, USA); hamster antimouse FasL mAb cross-reacting with rat FasL, clone MFL4 (32); mouse mAb anti-rat ICAM-l (IgGl, Seikagaku America Inc, Rockville, MA, USA); as a negative control, mouse mAb anti-human CDl6 (clone 3G8, IgG1); hamster anti-mouse Fas agonistic mAb (Jo-2, PharMingen); mouse anti-human Fas cross-reacting with rat Fas, clone 13 (Transduction Laboratories); mouse anti- $\alpha$-tubulin mAb (IgGl, Calbiochem, Cambridge, UK); and rabbit anti-von Willebrand factor (Dako, Glostrup, Denmark). A rabbit anti-human FLIP antibody (26) (kindly provided by Dr. K. Walsh, St. Elizabeth's Medical Center, Boston, MA, USA) and a rat anti-human FLIP $\mathrm{MAb}$ (clone Dave-2, cross-reacting with mouse FLIP) (Alexis, San Diego, CA, USA) were used to detect FLIP.

\section{Western Blot Analysis}

Cells were washed in PBS, trypsinated, and incubated in lysis buffer (0.1\% SDS, $1 \%$ NP40, $0.5 \%$ deoxycholic acid, 1\% PMSF and $10 \%$ aprotinin in $10 \mathrm{mM}$ Tris pH 7.4) (all from Sigma, St. Louis, MO, USA). Twenty micrograms of protein, measured using a BCA kit (Pierce, Rockford, IL, USA), were 
boiled for $3 \mathrm{~min}$ in SDS sample buffer and loaded onto $10-12 \%$ SDS-polyacrylamide gels followed by electrophoresis and blotting onto nitrocellulose membranes. Membranes were then blocked (overnight, $4{ }^{\circ} \mathrm{C}$ ) with PBS $/ 0.2 \%$ Tween-20/5\% nonfat dry milk for all the antibodies with the exception of the rabbit anti-FLIP antibody (blocked for $1 \mathrm{hr}$ at room temperature with PBS/0.2\% Tween-20/2\% nonfat dry milk). Membranes were then incubated ( $2 \mathrm{hr}$ at room temperature) with $1 \mu \mathrm{g} / \mathrm{ml}$ of the different antibodies using the same blocking solution. Membranes were then washed and incubated $(2 \mathrm{hr}$ at room temperature) with horseradish peroxidaselabeled secondary antibodies (Jackson Immunoresearch, West Grove, PA, USA) and detected with enhanced chemoluminiscence (Amersham, Freiburg, Germany) using x-ray films.

\section{Cytofluorometry and Immunocytology}

FasL was analyzed by cytofluorimetry and immunocytology on permeabilized or nonpermeabilized ECs. Culture of ECs was performed in the presence of $1 \mu \mathrm{g} / \mathrm{ml}$ of KB8301 (PharMingen), an inhibitor of metalloproteinases responsible for cleavage of FasL, which was added 12 and $1 \mathrm{hr}$ before analysis. Adherent cells were detached by a very brief incubation with trypsin $(0.05 \%)$ and EDTA $(0.02 \%)$ and immediately resuspended in culture medium. Permeabilization was obtained by incubating ECs $(20 \mathrm{~min}$ at room temperature) with a solution containing $2 \%$ paraformaldehyde (Merck, Darnstadt, Germany) and $0.5 \%$ saponin (Sigma) in PBS. With permeabilized cells, all subsequent solutions contained $0.1 \%$ saponin. For cytofluorometric analysis, ECs were incubated with $10 \mu \mathrm{g} / \mathrm{ml}$ of the different antibodies, washed, incubated with FITC-labeled secondary antibodies, washed, and fixed before analysis with a FACScalibur cytofluorometer (Becton Dickinson, Mountain View, CA, USA).

Immunostaining of isolated cells was performed on cells cultured with KB8301, transferred onto glass slides by cytocentrifugation $\left(2 \times 10^{4}\right.$ cells $)$, fixed and permeabilized. Cytospun cells were then hydrated with PBS, incubated with $0.3 \%$ hydrogen peroxide in methanol (1:10 vol:vol), and blocked with rat serum at $10 \%$ in PBS/BSA $1 \%$. Thereafter, cytocentrifuged cells were incubated with primary antibodies at $10 \mu \mathrm{g} / \mathrm{ml}$, washed, incubated with biotin-conjugated secondary antibodies, followed by horseradish peroxidase-conjugated streptavidin (Vector Laboratories, Burlingame, CA, USA), and then revealed with VIP substrate (Vector Laboratories). All incubations were performed at room temperature in a humid chamber.

\section{Cytotoxicity Assay}

The mouse Fas-sensitive cell line A20 was used to measure the killing activity of ECs. A20 cells $\left(10^{4}\right.$ cells) labeled with ${ }^{51} \mathrm{Cr}$ were incubated $(4 \mathrm{hr}$ at $37{ }^{\circ} \mathrm{C}$ ) in triplicates in round-bottom 96-well plates with increasing numbers of EC obtained from rat hearts. COS cells transduced with AdFasL and the anti-Fas Jo-2 mAb at $2.5 \mu \mathrm{g} / \mathrm{ml}$ were used as positive controls. When rat aortic ECs were used as targets, cells were labeled with ${ }^{51} \mathrm{Cr}$ and incubated $\left(4,6\right.$, and $19 \mathrm{hr}$ at $\left.37^{\circ} \mathrm{C}\right)$ in triplicates $\left(10^{4}\right.$ cells per well) with increasing numbers of L5178Y cells or L5178Y cells expressing rat or mouse FasL. ${ }^{51} \mathrm{Cr}$-labeled human Jurkat cells were used to evaluate the apoptosis mediated by rat FasL by incubating ( $4 \mathrm{hr}$ at $37^{\circ} \mathrm{C}$ ) with increasing numbers of COS cells transduced with AdFasL. ${ }^{51} \mathrm{Cr}$ release in the supernatant was measured using a scintillation counter and the percentage of specific cell lysis was calculated by the formula: $100 \times($ experimental cpm-cpm medium only) $/(100 \%$ lysis cpm-cpm medium only). One hundred percent lysis was obtained by incubating cells with $1 \%$ SDS.

To induce apoptosis by staurosporine or etoposide (diluted in DMSO), cells were plated overnight in triplicate in flat-bottom 96-well plates $\left(1.5 \times 10^{4}\right.$ cells per well) and cultured with the indicated concentrations of these compounds for $8 \mathrm{hr}$.

\section{Recombinant Adenovirus and Cell Transduction}

AdFasL contains an expression cassette with the human CMV promoter, an SV40 intron, the rat FasL CDNA, and SV40 polyadenylation sequences, and was constructed, propagated, and titered as previously described for other adenoviral vectors (34). AdlacZ coding for nls- $\beta$-galactosidase $(\beta$-Gal) (35) and $\mathrm{AdI} \kappa \mathrm{B}$ expressing $\mathrm{I} \kappa \mathrm{B} \alpha$ (36) (kindly provided by Dr. R. de Martin, Department of Vascular Biology, University of Vienna, Austria) have been previously described. Endothelial or COS cells $\left(2 \times 10^{5}\right.$ in 6-well plates) were incubated with adenoviruses at various multiplicity of infection (MOI) ranging from 1.5 to $500 \mathrm{PFU} / \mathrm{cell}$ at $37{ }^{\circ} \mathrm{C}$ for $90 \mathrm{~min}$ under agitation in $0.6 \mathrm{ml}$ of DMEM supplemented with $1 \%$ FCS. At the end of the incubation period, medium containing the adenoviruses was removed and medium supplemented with $10 \%$ FCS $(2 \mathrm{ml})$ was added. Cell viability was analyzed after removal of floating cells. Apoptosis and lacz expression was evaluated after $18 \mathrm{hr}$ of culture on floating and adherent cells pooled together.

\section{DNA Constructs and Plasmid Transfection}

In the pCMVFasL and pCMVlacZ plasmids, rat FasL cDNA (kindly provided by S. Nagata, Osaka University, Osaka, Japan) or Escherichia coli $\beta$-Gal coding sequences were placed under the control of the human cytomegalovirus (CMV) promoter. The pCMVFLIP plasmid coding for the long isoform of mouse FLIP $\left(\right.$ FLIP $\left._{L}\right)$ under the control of the human CMV promoter was kindly provided by Dr. Y. Pewzner-Jung (Rockefeller University, NY, USA). Endotoxin-free plasmids for EC transfection were purified using the EndoFree kit (Qiagen, Hilden, Germany). Rat aortic ECs $\left(2 \times 10^{5}\right.$ in 6-well plates $)$ 
were plated overnight and transfected using the Superfect transfection kit (Qiagen) or Lipofectamine (Gibco, Cergy Pontoise, France). Cotransfection of rat aortic ECs was performed using $0.33 \mu \mathrm{g} /$ well of pCMVFasL together with $0.66 \mu \mathrm{g} /$ well of either pCMVlacZ or pCMVFLIP. Cell viability, apoptosis and $\beta$-Gal expression were evaluated after 24 or $48 \mathrm{hr}$ of culture.

Detection of $\beta$-Gal and Apoptosis in Adenovirus-Transduced or Transfected Cells

$\beta$-Gal expression was detected by X-Gal staining. Apoptosis was detected in cytospun cells fixed with paraformaldehyde using both the TdT-mediated dUTP nick-end labeling (TUNEL) technique with the ApopTag kit (Oncor, Gaithersburg, MD, USA) following the manufacturer's instructions and by FACS analysis of cells stained with FITC-labeled annexin V (PharMingen). Cell viability was evaluated using a (3-[4,5 dimethylthiazol-2-yl $] 2,5-$ diphenyltetrazolium bromide) (MTT) (Sigma) viability assay. To generate a standard curve of viable cells, a graded number of cells $(100,50,25$, and $12.5 \%$ of the original concentration) were plated and cultured overnight. Cells incubated with water provided $100 \%$ lysis. Cell viability was evaluated by incubating $\left(3 \mathrm{hr}\right.$ at $\left.37^{\circ} \mathrm{C}\right)$ the cells with a solution of $1 \mathrm{mg} / \mathrm{ml}$ of MTT in culture medium. The solution was then removed and MTT crystals were dissolved by adding $100 \mu \mathrm{l}$ of DMSO. Evaluation of apoptosis after FasL and/or FLIP F $_{\mathrm{L}}$ transfection of rat aortic ECs in 6-well plates was performed by adding $1.5 \mathrm{ml}$ of MTT and $1.5 \mathrm{ml}$ of DMSO per well. One-hundred microliters of the MTT dissolved crystals were then loaded in triplicates in flat-bottom 96-well plates and absorbance was evaluated at $550 \mathrm{~nm}$. The number of viable cells was calculated by plotting DO values obtained in experimental conditions against those obtained with graded numbers of cells. For transfection experiments, results are reported as the percentage of viable cells for each plasmid versus pCMVlacZ taken as a control of $100 \%$ viability $(100 \times$ experimental plasmid viable cells/pCMVlacZ viable cells). For experiments of induction of apoptosis with staurosporine, etoposide, or AdFasL, results are reported as the percentage of viable cells versus culture in the presence of $0.2 \%$ DMSO $(100 \times$ experimental condition viable cells/0.2\% DMSO viable cells).

\section{In Situ Adenoviral Transduction of Endothelium and Analysis of Apoptosis}

Efficient transduction of quiescent endothelium with adenoviruses was obtained using a technique previously described (37). Briefly, abdominal aortas were excised from male Sprague-Dawley rats, opened longitudinally, cut into pieces of $3 \times 4 \mathrm{~mm}$, transduced with $10^{10}$ PFU of adenoviruses and cultured $\left(36 \mathrm{hr}\right.$ at $\left.37^{\circ} \mathrm{C}, 5 \% \mathrm{CO}_{2}\right)$ with the endothelial face facing the top in DMEM supplemented with
$10 \%$ FCS and $1 \mathrm{mM}$ of glutamine. Pieces of aorta were then analyzed using X-Gal or biotinylated annexin $V$, revealed with streptavidin-peroxydase and VIP substrate, and observed with a conventional microscope.

\section{Results}

\section{Rat EC Express Predominantly Intracellular FasL}

To overexpress cell membrane FasL on rat ECs we first generated transgenic rats (38) using the ICAM-2 endothelial-specific promoter (previously used to generate transgenic rats [30]) and a tetracyclineinducible system. All rat transgenic lines for FasL (six lines) showed mRNA for transgene-derived cell membrane FasL with some control by the tetracycline system (data not shown). Nevertheless, even in the lines with higher mRNA levels, FasL expression was low because it was detectable at the mRNA level only after RT-PCR amplification (38) and was not detectable at the protein level (data not shown).

Despite human ECs having been described as being resistant to Fas-mediated apoptosis and expression of cell-surface FasL (20), we hypothesized that failure to obtain transgenic rats expressing significant levels of cell membrane FasL could be due to uterine loss of animals expressing FasL due to leaky expression of the tetracycline-inducible system and sensitivity of rat ECs to FasL-mediated apoptosis.

Western blot analysis of rat aortic ECs (Fig. 1A) and human ECs (data not shown) with anti-FasL $\mathrm{mAb}$ showed the presence of a single band of the expected molecular weight $(38 \mathrm{kDa})$ that had the same gel mobility as FasL from activated Jurkat cells, whereas, as previously described (39), smooth muscle cells were negative (Fig. 1A). Rat heart ECs were also positive for FasL expression (data not shown).

We questioned whether rat ECs express FasL on the cell surface and because intracellular FasL has been described in monocytes (12), NK cells, and CTLs (13), we also analyzed this cellular compartment for FasL expression. Nonpermeabilized primary rat ECs obtained from hearts or aortas showed very low or undetectable levels of cell-surface FasL, as assessed by FACS analysis with two different anti-FasL mAbs (clones 33 and G247-4) (Fig. 1B). When permeabilized ECs were analyzed, both types of EC were shown to be positive with both antiFasL mAbs (Fig. 1B). As control antibodies, isotype control $3 \mathrm{G} 8 \mathrm{mAb}$ was negative for cell-surface and intracellular staining, the anti-ICAM-1 mAb was positive only for cell-surface staining, and the antivWF antibody showed only intracellular reactivity (Fig. 1B). Permeabilized HL-60 and Jurkat cells were used as negative and positive cellular controls of intracellular staining for FasL, respectively (data not shown).

Predominant intracellular expression of FasL on rat ECs was confirmed morphologically on cytospun 


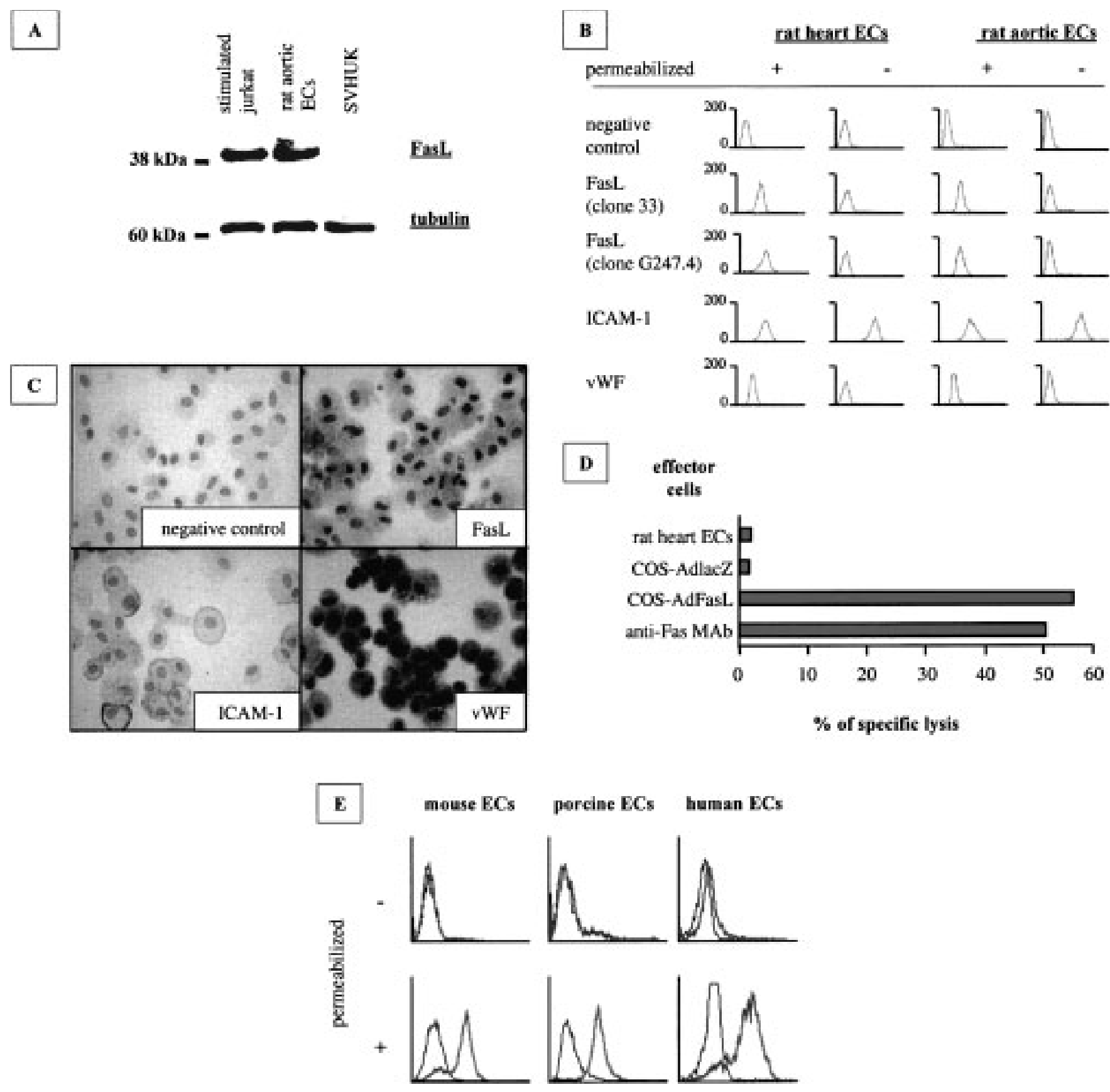

Fig. 1. Rat ECs express predominantly intracellular FasL. Rat ECs from hearts or aortas were cultured in the presence of the metalloproteinase inhibitor KB8301. (A) Western blot analysis of rat aortic ECs using an anti-FasL MAb (clone 33) and an anti- $\alpha$-tubulin MAb as loading control. Jurkat-stimulated cells and SVHUK smooth muscle cells were used as positive and negative controls, respectively. (B) For FACS analysis, cells were either permeabilized or not for intracellular or cell-surface staining respectively with antibodies: negative control (3G8); anti-FasL (clones 33 and G247-4); anti-ICAM-1 and anti-von Willebrand factor (vWF). (C) ECs from hearts were cytospun, permeabilized, and analyzed with the indicated antibodies. ECs show no staining with the isotype control mAb (3G8), intracellular staining with both anti-FasL mAbs (clone G247-4 in this figure), and the anti-vWF antibody, and cell membrane staining with the anti-ICAM-1 mAb. (D) Cytotoxicity assay using apoptosis-sensitive ${ }^{51} \mathrm{Cr}$-labeled A20 cells as targets and rat heart ECs or COS cells as effectors (100 to 1 target to effector ratio). COS cells were transduced with AdlacZ or AdFasL

(100 PFU/cell), cultured overnight, and then used as negative and positive controls, respectively. The agonistic anti-Fas mAb Jo-2 was used as an additional positive control. (E) FACS analysis of FasL expression on human, mouse, and pig ECs permeabilized or not for intracellular or cell-surface staining respectively with antibodies: negative control (3G8, thin histograms); anti-FasL (clone G247-4, thick histograms). Results are representative of four experiments. 
and permeabilized cells. Rat EC FasL reactivity was detected intracellularly with clone 247-4 (Fig. 1C) and 33 (data not shown) anti-FasL mAbs, as well as for vWF used as an intracytoplasmic control, whereas ICAM-1 was detected on the cell-surface (Fig. 1C).

To test for the presence of functional cell-surface FasL on rat ECs, we used A20 cells as targets and rat ECs as effectors in cytotoxicity assays. A20 cells incubated with heart EC or aortic rat EC were not killed, whereas A20 cells incubated with an agonistic anti-Fas mAb or with COS cells transduced with AdFasL but not with AdlacZ showed over $50 \%$ specific lysis (Fig. 1D), as previously described (31). The absence of detectable apoptosis against A20 cells was also confirmed using rat ECs, which were left adherent prior to and during the cytotoxicity assay (data not shown), indicating that absence of FasL could not be explained by protein digestion during cell harvesting using trypsin.

FACS analysis of FasL expression on ECs of other species showed that in human, mouse, and porcine ECs, the large majority of FasL was also expressed intracellularly and that cell-surface expression was low (in human EC) or undetectable (in mouse and porcine EC) as analyzed with both antiFasL mAbs (Fig. 1E for clone G-247-4 and data not shown for clone 33).

These results indicate that FasL present in rat ECs from both microvascular and large vessels is exclusively expressed intracellularly. Expression of FasL in ECs from other species is also largely predominant within ECs, although low cell-surface expression was detectable in human cells.

\section{Rat EC Die by Fas-Dependent Apoptosis Following Expression of Cell-Surface FasL}

Because transgenic rats expressing cell membrane FasL could not be obtained and rat ECs expressed FasL intracellularly, we asked whether cell surface overexpression of FasL would result in rat EC apoptosis.

Rat EC transduction with AdlacZ at $100 \mathrm{PFU} / \mathrm{cell}$ resulted in $60-80 \%$ of $\beta$-Gal + cells (data not shown) and no cell death (Fig. 2A), whereas rat EC transduction with the same dose of AdFasL showed a large majority of cells with characteristics of dead cells (Fig. 2B), which was confirmed by their uptake of eosin (data not shown). TUNEL analysis of AdlacZ (Fig. 2C) and AdFasL-transduced (Fig. 2D) cells confirmed the apoptosis of rat ECs only after AdFasL transduction. Annexin- $V$ staining of AdlacZ-transduced cells showed no increase in apoptotic cells compared to nontransduced cells (Fig. 2E), whereas apoptosis was confirmed in $60-90 \%$ of AdFasL-transduced cells (Fig. 2F). Incubation with a neutralizing anti-FasL mAb confirmed that apoptosis was FasL-dependent because it reduced labeling with annexin V (Fig. 2F) almost to nontreated control levels and increased cell viability (data not shown). Apoptosis of rat ECs after adenovirus-mediated membrane expression of FasL was dose-dependent; incubation of rat ECs with 1.5, 3, 6, 12, 25, 50 and 100 PFU of AdFasL resulted in 5, 15, 18, 30, 37, 38
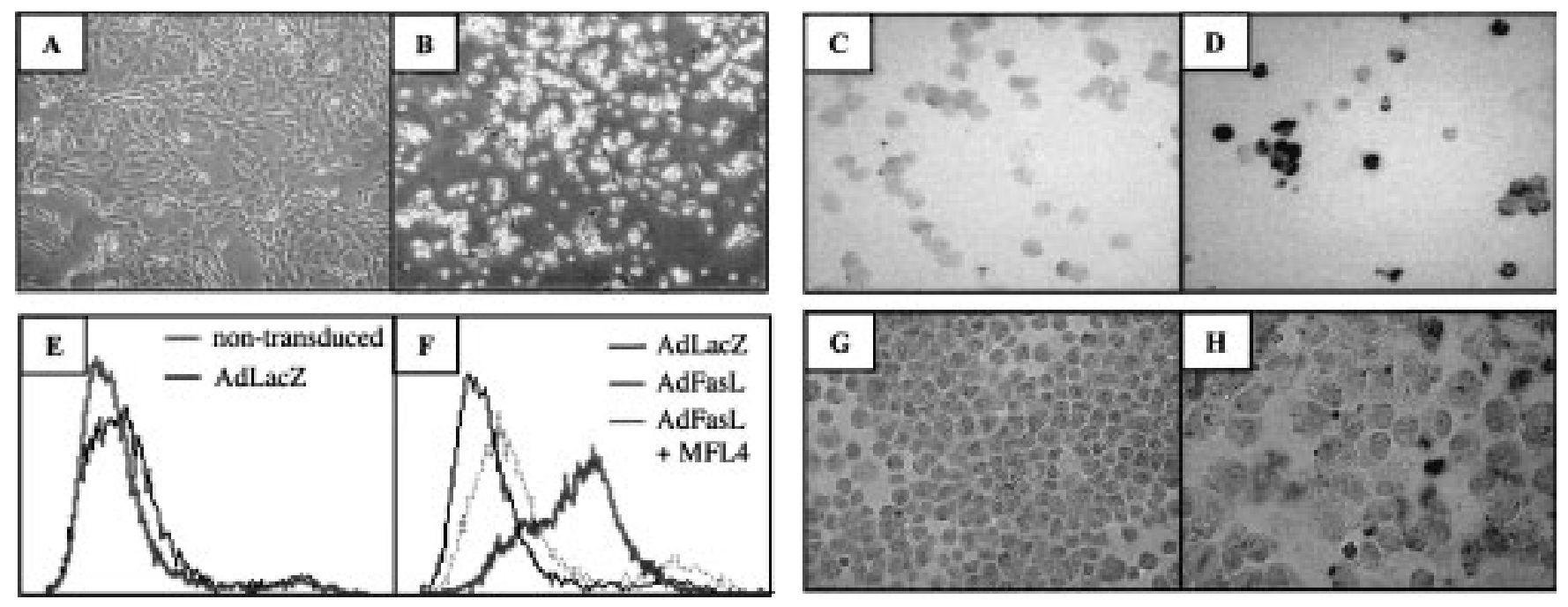

Fig. 2. Apoptosis of rat ECs after expression of membrane FasL. Rat aortic ECs were transduced with AdlacZ or AdFasL (100 PFU/cell), cultured for $18 \mathrm{hr}$, and analyzed for apoptosis. Phase contrast microscopic analysis of (A) AdlacZ-transduced cells showed adherent cells with morphology of viable cells, whereas (B) a majority of AdFasL-transduced cells were detached and with poor viability. The TUNEL assay showed (C) no apoptotic cells after transduction with AdlacZ, whereas (D) most cells were TUNEL-positive after AdFasL transduction. Annexin V staining showed (E) no apoptotic cells among AdlacZ-transduced cells and (F) $>75 \%$ of cells positive after transduction with AdFasL. Overnight incubation with a blocking anti-FasL mAb (MFL4 at $25 \mu \mathrm{g} / \mathrm{ml}$ ) drastically reduced the percentage of apoptotic cells (F). The TUNEL assay on rat aortic ECs transiently transfected with (G) pCMVlacZ did not show apoptotic cells, whereas $(\mathbf{H})$ transfection with phCMVFasL resulted in apoptosis as seen by the presence of TUNEL-positive cells. Results are representative of three experiments. 
and $47 \%$ of annexin V-positive cells, respectively. Apoptosis was also time-dependent because culture of rat ECs for 2, 4, 8 and $18 \mathrm{hr}$ with AdFasL (MOI of $100 \mathrm{PFU}$ ) resulted in 3, 9, 39 and $63 \%$ of annexin V-positive cells, respectively. Transduction of heartderived primary rat ECs with AdFasL also resulted in massive apoptosis (data not shown). COS cells transduced under the same conditions (and up to 500 PFU) showed functional cell-surface FasL expression (Fig. 1D) and no cell death (data not shown), excluding a nonspecific toxicity in the AdFasL preparation that killed rat EC. To exclude potential pro-apoptotic signals due to adenovirus transduction of EC that could cooperate with FasL in the induction of apoptosis, FasL was also expressed by transient plasmid transfection. TUNEL assays did not reveal significant numbers of apoptotic cells after transfection of rat ECs with pCMVlacZ (Fig. 2G) (which resulted in approximately $5 \%$ of Xgal+ cells), whereas transient transfection with the phCMVFasL plasmid resulted in detection of a comparable percentage of apoptotic cells (Fig. 2H).

Thus, rat ECs die by apoptosis after overexpression of FasL and this apoptosis is dependent on the interaction of FasL with cell surface Fas.

\section{EC of Mouse but not of Human or Porcine Origin Die by Apoptosis Following Expression of Membrane FasL}

Published results concerning the sensitivity of ECs from different species to Fas-mediated apoptosis are contradictory. Human ECs in the resting state have been previously reported to be resistant to Fasmediated apoptosis $(20,22)$ although human capillary ECs were shown to be sensitive (25). On the other hand, mouse ECs were reported to die by apoptosis in vitro (21) and in vivo (19).
Therefore, we compared the apoptosis of ECs from different species following transduction with AdFasL. Preliminary experiments enabled the definition of the MOI for AdFasL and the time of culture that would result in optimal expression of FasL in the different EC types. Rat and mouse ECs expressed lower levels of FasL (even at MOI of 500, data not shown) compared to human and porcine ECs (Fig. 3A). Expression levels of FasL did not correlate with induction of apoptosis because rat and mouse ECs showed high levels of apoptosis $(>75 \%)$, whereas human and pig ECs displayed no apoptosis $(<2 \%)$ as detected by binding of annexin $\mathrm{V}$ (Fig. 3A). Transduction of ECs from these different species with AdlacZ at an MOI of 100 resulted in $>75 \%$ transduction as evaluated by X-Gal staining (data not shown). Lower expression levels of FasL in rat and mouse ECs probably reflects poor viability due to ongoing apoptosis.

Western blot analysis of Fas expression by ECs from different species revealed a band of expected molecular weight $(45 \mathrm{kDa})$ comparable to that of Jurkat used as positive control in ECs of human, porcine, and rat origin (Fig. 3B). Therefore, resistance to FasL-mediated apoptosis by ECs from porcine or human origin and sensitivity by rat ECs cannot be explained by absence or higher expression of Fas, respectively.

Although mouse FasL is able to mediate apoptosis of human cells and therefore does not show species restriction (40), we analyzed whether rat FasL was also capable of inducing apoptosis of human cells. COS cells expressing rat FasL after transduction with AdFasL-induced apoptosis of human Jurkat cells $(41.18 \pm 2 \%)$, whereas AdlacZ-transduced

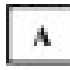

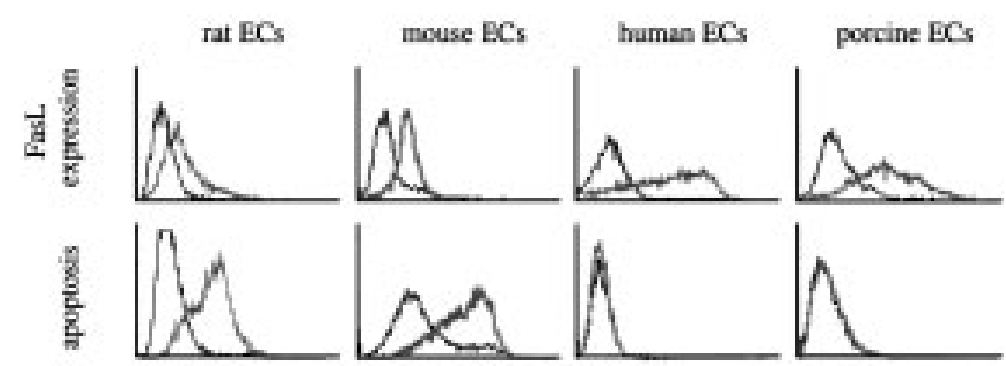

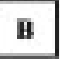

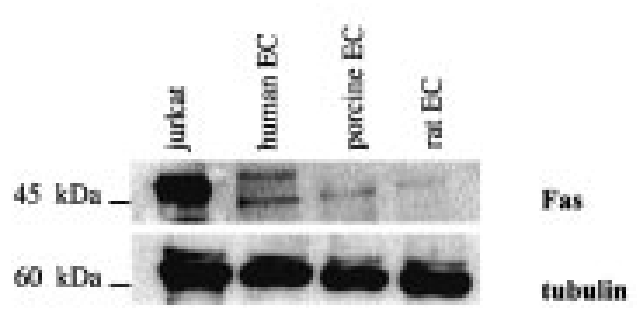

Fig. 3. Analysis of Fas, FasL, and apoptosis in ECs from different species. (A) ECs of rat, mouse, human, and pig origin were transduced with AdlacZ (thin histograms) or AdFasL (thick histograms) with an MOI of 100 for rat aortic and mouse ECs and 500 for human and pig ECs. Cells were then cultured for $18 \mathrm{hr}$ for rat aortic and mouse ECs and $72 \mathrm{hr}$ for human and pig ECs. Cells were then analyzed by FACS using an anti-FasL mAb (clone MFL4) or FITC-labeled annexin V. (B) Western blot analysis of Fas expression (clone $13 \mathrm{mAb}$ ) on Jurkat cells used as positive control and on human, porcine and rat ECs. $\alpha$-Tubulin was used as loading control. Results are representative of three experiments. 
Table 1. FasL induces time- and dose-dependent apoptosis of rat aortic ECs

Target Cells

\begin{tabular}{|c|c|c|c|c|c|c|c|}
\hline \multirow[b]{3}{*}{ Effector Cells } & \multirow[b]{3}{*}{ Effector:Target Ratios } & \multirow{2}{*}{\multicolumn{3}{|c|}{ Rat Aortic ECs }} & & & \\
\hline & & & & & \multicolumn{3}{|c|}{ A20 } \\
\hline & & $4 \mathrm{hr}$ & $6 \mathrm{hr}$ & $19 \mathrm{hr}$ & $4 \mathrm{hr}$ & $6 \mathrm{hr}$ & $19 \mathrm{hr}$ \\
\hline \multirow[t]{4}{*}{ L5178Y-rat FasL } & 1 & $<1^{*}$ & $<1$ & $10.0 \pm 2.7$ & $24.0 \pm 1.6$ & $32.3 \pm 1.5$ & $58.8 \pm 0.3$ \\
\hline & 10 & $<1$ & $<1$ & $27.7 \pm 1.8$ & $38.3 \pm 1.0$ & $40.0 \pm 2.3$ & $56.2 \pm 3.4$ \\
\hline & 50 & $1.0 \pm 0.4$ & $1.1 \pm 0.4$ & $47.5 \pm 5.3$ & $39.6 \pm 1.0$ & $39.6 \pm 1.7$ & $70.0 \pm 0.4$ \\
\hline & 100 & $1.6 \pm 0.3$ & $2.6 \pm 1.1$ & $59.0 \pm 4.5$ & $36.8 \pm 1.0$ & $39.5 \pm 1.5$ & $76.5 \pm 3.0$ \\
\hline \multirow[t]{4}{*}{ L5 178Y } & 1 & $<1$ & $<1$ & $<1$ & $<1$ & $<1$ & $<1$ \\
\hline & 10 & $<1$ & $<1$ & $<1$ & $<1$ & $<1$ & $<1$ \\
\hline & 50 & $<1$ & $<1$ & $<1$ & $<1$ & $<1$ & $<1$ \\
\hline & 100 & $<1$ & $<1$ & $<1$ & $<1$ & $<1$ & $12.4 \pm 2.7$ \\
\hline
\end{tabular}

Rat aortic ECs and A20 cells were labeled with ${ }^{51} \mathrm{Cr}$, seeded in 96 -well plates $\left(10^{4}\right.$ cells/well) and incubated with increasing ratios of L5178Y effector cells expressing or not cell-surface rat FasL for the indicated times.

*Results are expressed as the percentage of specific cell lysis.

COS cells did not $(0.5 \pm 0.6 \%)(12.5$ to 1 ratio). Thus, apoptosis of rat and mouse ECs and not of human or pig ECs by rat FasL was not due to species restriction.

We then tested whether rat ECs were sensitive to Fas-mediated apoptosis by FasL-expressing cells, a situation that could mimic leukocyte-mediated EC apoptosis during inflammatory conditions of the vessel wall. Rat aortic ECs incubated with increasing numbers of L5178Y effector cells expressing rat FasL (Table 1), or mouse FasL (data not shown), showed a time- and dose-dependent increase in cell death, whereas incubation with nontransduced L5178Y cells resulted in no loss of cell viability (Table 1). Rat aortic EC death induced by Fas-FasL interaction required a longer time and higher cell effector-to-target ratio compared to the A20 apoptosis-sensitive cells used as a positive control (Table 1).

In conclusion, resting rat and mouse ECs but not human and pig ECs die by apoptosis following expression of cell-surface FasL or interaction with FasL expressed by other cells.

\section{Rat and Mouse ECs are Equally Sensitive as Human or} Porcine EC to Staurosporine or Etoposide-Induced Apoptosis

We then asked whether rat or mouse ECs are more sensitive to other inducers of apoptosis compared to human or pig ECs. Incubation with staurosporine or etoposide resulted in a comparable decrease in cell viability in all EC types, whereas transduction with AdFasL resulted in decreased viability only in rat and mouse ECs (Fig. 4). Thus, rat aortic ECs and mouse ECs showed increased sensitivity to Fas-induced apoptosis but not to other apoptotic stimuli.

\section{In Situ Transduction of Resting Endothelium also Results in Apoptosis}

Because ECs lining the vessels are essentially nondividing cells and receive multiple signals from underlying basement membranes and cells, in vitro cultured ECs may not readily reflect the sensitivity of normal ECs to Fas-mediated apoptosis. Therefore, we analyzed whether in situ transduction of rat endothelium with AdFasL would also trigger EC apoptosis. Incubation with AdlacZ used as a positive control of resting endothelium transduction resulted in numerous X-Gal-positive ECs (Fig. 5A). Analysis of AdlacZ-transduced aortas, used as a negative control, showed no EC apoptosis as determined by annexin $\mathrm{V}$ binding (Fig. 5B). As a positive control, transduction with AdI $\kappa$ B followed by culture with TNF- $\alpha$ resulted in widespread EC apoptosis (Fig. 5C). Transduction of aortas with AdFasL resulted in massive apoptosis of ECs (Fig. 5D).

These results indicate that FasL expression in rat ECs within normal quiescent endothelium also results in apoptosis.

\section{FLIP Expression is Reduced in Rat and Mouse ECs Compared to Human ECs}

FLIP expression levels correlate with the sensitivity of human ECs to apoptosis through Fas $(24,26)$. Therefore, we analyzed whether increased sensitivity to Fas-mediated apoptosis in rat and mouse ECs compared to human ECs could be associated with lower levels of FLIP. Western blot analysis with an anti-human FLIP polyclonal antibody revealed that human ECs (Fig. 6) and porcine ECs (data not shown) expressed the long (55-kDa) and short (25kDa) forms of FLIP (FLIP (F) $_{\text {FLIP }}$, respectively) and that FLIP $_{L}$ was more represented than FLIP $_{S}$. 


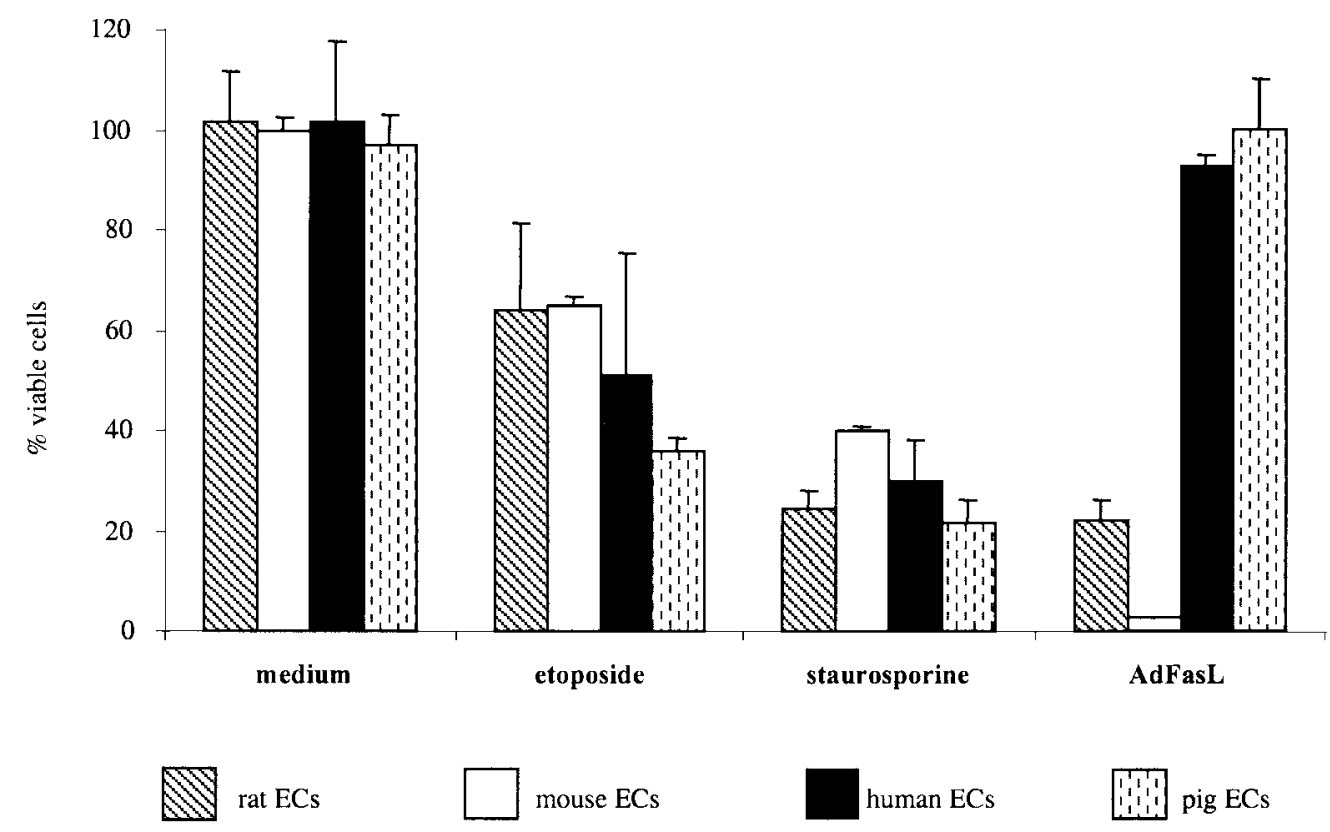

Fig. 4. ECs from different species show comparable sensitivity to apoptosis induced by etoposide or staurosporine. EC of rat (stippled histograms), mouse (white histograms), human (black histograms), and pig (dotted histograms) origin were incubated for 8 hr with etoposide $(200 \mu \mathrm{M})$ or staurosporine $(200 \mathrm{nM})$. Rat aortic and mouse ECs were transduced with 100 MOI of AdFasL and cultured for $16 \mathrm{hr}$, and human and porcine ECs were transduced with 500 MOI and cultured for $48 \mathrm{hr}$. Cell viability was analyzed using an MTT assay. Numbers of viable cells were plotted against numbers of viable cells cultured with medium and DMSO $(0.2 \%)$ or after transduction with AdlacZ (100\% of viable cells). Results are expressed as the mean \pm SD of triplicates from one experiment representative of three performed under the same conditions.

Human ECs expressed much higher levels of FLIP compared to rat and mouse ECs, whereas FLIP $\mathrm{S}$ was expressed at comparable levels in all types of ECs (Fig. 6). Recognition of mouse FLIP $_{\mathrm{L}}$ in COS cells transfected with a mouse FLIP $_{L}$ expression plasmid (Fig. 6) and comparable levels of FLIP $_{S}$ expression enabled the confirmation that this anti-human FLIP antibody crossreacts with FLIP from other species and that low levels of FLIP $_{L}$ in mouse and rat ECs is not due to lack of crossreactivity. Furthermore, Western blot analysis using an anti-human FLIP monoclonal antibody (clone Dave-2) also showed the presence of FLIP $_{\mathrm{L}}$ in human and pig but not mouse ECs (data not shown). Therefore, although we cannot formally exclude that these antibodies raised against human FLIP have a lower reactivity with FLIP from other species, an increased sensitivity of rat and mouse ECs to Fas-mediated apoptosis was correlated with lower expression of FLIP ${ }_{L}$.

Increased Expression of FLIP by Rat EC Augmented their Resistance to Apoptosis Induced by Expression of FasL on the Cell-Surface Membrane

To test whether an increase in FLIP $_{\mathrm{L}}$ levels in rat ECs could protect these cells from Fas-mediated apoptosis, we simultaneously expressed FasL and FLIP $_{L}$. Expression of FLIP ${ }_{L}$ by rat ECs after transfection with pCMVFLIP was confirmed by Western blot (Fig. 7A). Cotransfection with plasmids coding for FasL and lacZ resulted in a significant decrease in the percentage of viable cells compared to cells transfected with lacZ alone $(65.3 \pm 8.1$ versus $100 \%$, respectively, $p<0.05, n=4$ ) (Fig. 7B). The lower percentages of apoptosis observed after transfection compared to cultures transduced with AdFasL likely reflects the lower efficiency of DNA incorporation into EC using transfection versus adenoviral vectors. In contrast, cotransfection with plasmids coding for FasL and FLIP resulted in partial protection against apoptosis because percentages of viable cells were comparable to those of cells transfected with lacZ alone $(84.1 \pm 11.1 \%$, versus $100 \%$, respectively, $p>0.05$, $n=4)$ (Fig. 7B). As a control, cotransfection with plasmids coding for lacZ and FLIP yielded percentages of viable cells comparable to lacZ alone $(95.5 \pm 6.3 \%$, versus $100 \%$, respectively, $p>0.05, n=4$ ) (Fig. 7B).

These results suggest that constitutive low levels of FLIP in rat ECs are responsible for rat EC sensitivity to apoptosis induced by Fas-FasL interaction, but it is possible that other mechanisms may also contribute to this phenomenon.

\section{Discussion}

Human ECs have been shown to express low levels of cell membrane FasL and TNF- $\alpha$ has been shown to down-regulate its expression and result in tissue infiltration by leukocytes (20). In contrast, mouse ECs showed absence of FasL cell-surface expression (21). FasL has also been shown to be preformed and stored in the cytoplasm of monocytes (12), NK cells, 


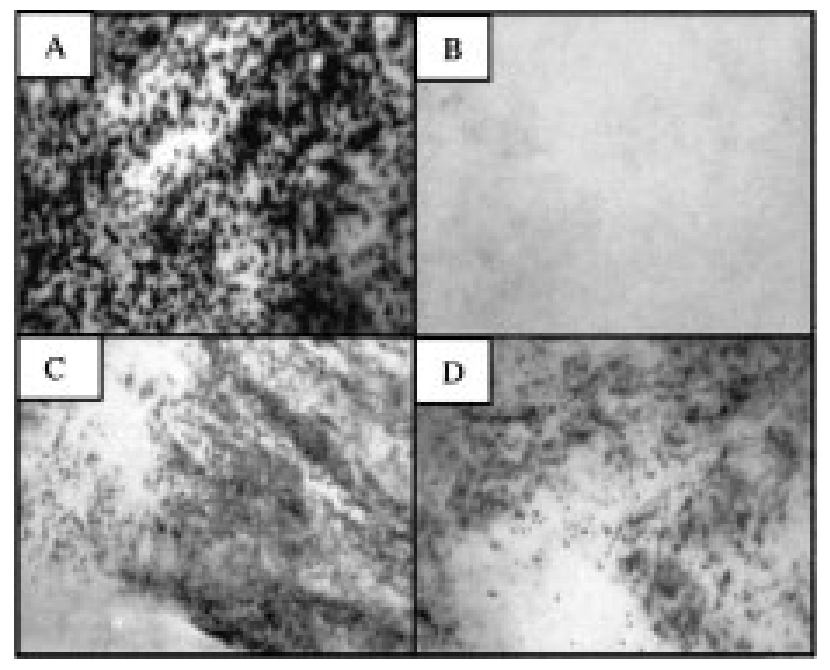

Fig. 5. FasL expression in rat quiescent endothelium results in apoptosis. Rat aortas were harvested and transduced in vitro with recombinant adenoviruses, cultured for $36 \mathrm{hr}$, incubated in X-Gal, or analyzed using annexin $\mathrm{V}$ and observed with a conventional microscope $(\times 100)$. The endothelial surface is shown facing the top. (A) AdlacZ, followed by incubation with X-Gal; (B) AdlacZ; (C) AdI $\kappa$ B and TNF- $\alpha$; and (D) AdFasL, followed by incubation with biotinylated annexin $\mathrm{V}$ and revealed with streptavidin-peroxydase and VIP substrate. Results are representative of three experiments performed under the same conditions.

and CTLs (13), but intracellular expression of FasL in ECs has not been previously reported. The susceptibility of rat and mouse ECs to FasL-mediated apoptosis supports our results showing absence of detectable cell-surface FasL on these cells.

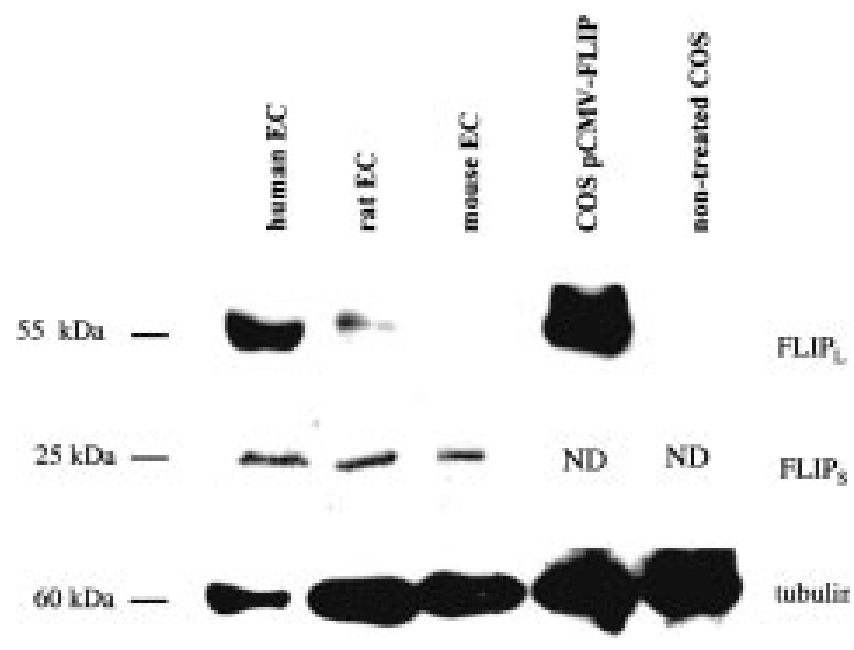

Fig. 6. Rat and mouse ECs express lower levels of FLIP compared to human ECs. Western blot analysis of FLIP in ECs of rat, mouse, or human origin and COS cells transfected or not with a plasmid encoding for mouse FLIP $_{L}$ (pCMVFLIP). FLIP was recognized using a rabbit anti-human FLIP that recognizes both FLIP $_{L}$ and FLIP . Comparable loading in each line was confirmed using an anti- $\alpha$-tubulin mAb. ND, not done. Results are representative of three experiments performed under the same conditions.
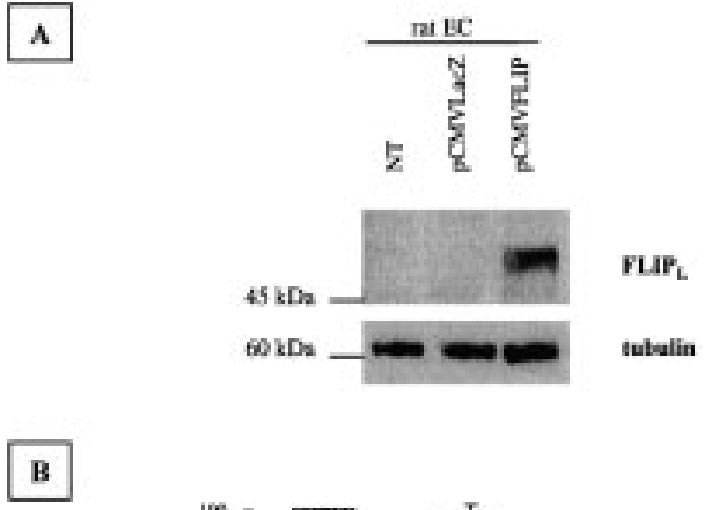

B

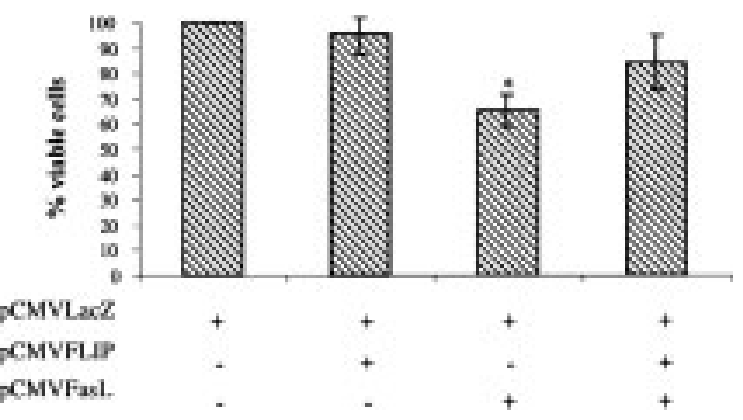

Fig. 7. Transfection of FLIP into rat ECs increases resistance to apoptosis induced by membrane expression of FasL. Rat aortic ECs were transfected with plasmids coding for $\beta$-Gal (pCMVlacZ), rat FasL (pCMVFasL), or mouse FLIP $_{\mathrm{L}}$ (pCMVFLIP). (A) Western blot analysis of FLIP expression (MAb clone Dave-2). NT, nontransfected. Detection of $\alpha$-tubulin was used as loading control. (B) Cell viability was analyzed using an MTT assay. Numbers of transfected viable cells were plotted against numbers of viable cells after transfection with pCMVlacZ alone ( $100 \%$ of viable cells). The results are expressed as \% mean $\pm \mathrm{SE}$ of viable cells from four experiments performed in duplicate for each experimental condition. ${ }^{*} p<0.05$ versus $\mathrm{pCMVlacZ}$ alone.

ECs of mouse $(17,21)$ and human origin (20) have been shown to express low levels of Fas and the present report shows expression of functional Fas in rat ECs; FasL-L5 178Y cells and FasL expression by ECs induced their apoptosis. Although resting human ECs from large vessels have been shown to be resistant to apoptosis mediated by anti-Fas or AdFasL transduction (20,39), ECs from subretinal capillaries were sensitive (25). Furthermore, incubation with oxidized lipoproteins or absence of matrix attachment leads to apoptosis of human ECs via Fas $(24,26,27)$. Importantly, and similarly to rat ECs, resting mouse ECs were killed by Fas ligation using agonistic anti-Fas antibodies $(19,21)$ or soluble FasL (19) in vivo. Furthermore, ECs from the liver and spleen but not from the lung are damaged by a Fas-mediated mechanism in mice with IL-2-induced vascular leak syndrome (17). Additionally, resting human microvascular ECs but not aortic human ECs showed Fas-mediated apoptosis induced by plasma from patients with thrombotic thrombocytopenic purpura (16). Therefore, there is heterogeneity in Fas-mediated 
EC apoptosis depending on the activation state, and on the species and organ origin of the ECs.

An adenovirus coding for mouse FasL has been used to reduce chronic rejection in a rat aortic allotransplantation model (41). This is in apparent contradiction with our results, but in the previous study transduced aortas were transplanted in an allogeneic combination and analysis of apoptosis in rat ECs after transduction were not reported.

Resting human ECs have been shown to express only FLIP $_{L}$ and not FLIP in most $(26,27,42)$ but not all previous publications (24). Induction of Fasmediated apoptosis in human ECs cultured with oxidized low-density lipoprotein $(26,27)$ or in the absence of matrix attachment (24) was associated with

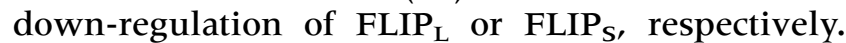
Protection against apoptosis was augmented via increased FLIP ${ }_{L}$ expression $(26,27,42)$ and was associated with inhibition of caspase-8 activation (27). FLIP $_{L}$ seems to be a more potent inhibitor of apoptosis than FLIP $_{S}(43)$. The present results in rat and mouse ECs show that sensitivity to apoptosis was dependent on FLIP $_{\mathrm{L}}$ levels; these cells expressed much lower levels compared to human ECs and showed increased resistance to Fas-mediated apoptosis after FLIP ${ }_{\mathrm{L}}$ overexpression. Results on the regulation of FLIP levels in human ECs are contradictory. One study showed positive regulation by the phosphatidylinositol 3'-kinase and the serine/threonine kinase Akt pathway and not by the mitogen-activated protein kinase/Erk cascade (27), whereas another study showed the opposite (24). The relative role of these two pathways in regulating FLIP levels in rat and mouse ECs compared to human ECs is presently under analysis.

Transgenic mice expressing FasL in $\beta$ cells of pancreatic islets (10), lymphocytes (44), cardiomyocytes (11), and thyroid epithelial cells (45) have been described but no reports are available on transgenic animals for FasL expressed on ECs. Depending on the level of FasL expression, several $(10,11)$ but not all (45) of these models showed cell apoptosis, leukocyte infiltration, and tissue lesions. The apoptosis of rat ECs expressing cell-surface FasL either in vitro or in situ after adenovirus- or plasmid-mediated expression, as well as in vivo studies in mice, suggests that the lack of transgenic rats expressing relevant levels of cell-surface FasL is due to apoptosis of ECs in vivo, probably resulting in nonviable animals. Previous results suggested (28) and our results demonstrate that pig aortic ECs are not killed after expression of FasL in vitro. On the one hand this suggests that the generation of pigs transgenic for FasL expressed in the endothelium could be a feasible and relevant strategy for xenotransplantation into humans. On the other hand, the fact that the activation state as well as the species and tissue origin (macrovascular versus microvascular ECs) of ECs modulates their sensitivity to apoptosis induced by Fas-FasL interaction also indicates that the result of overexpression of FasL by EC of pig organs is difficult to predict.

In conclusion, these results show species differences on the sensitivity of ECs to apoptosis by the Fas-FasL system depending on expression of membrane FasL and levels of FLIP $_{\mathrm{L}}$. Therefore, the apoptotic effect on ECs of FasL expressed on the cell membrane by leukocytes or by ECs after genetic engineering and the role of Fas-FasL interactions in vascular inflammatory conditions will have different outcomes depending on the activation state, vascular territory, and species in which these studies are performed.

\section{Acknowledgments}

This work was financed in part by the Groupement d'Intérêt Public INSERM-Aventis, the Fondation Transvie, and EEC grant Biomed2 BMM-CT983277. We are grateful to Regis Josien for critically reading the manuscript, to Valia Proust and Emmanuel Merieau for animal care, and to researchers that contributed reagents. We thank the Vector Core of the University Hospital of Nantes, supported by the Association Française contre les Myopathies (AFM), for producing the adenoviral vectors used in this study.

\section{References}

1. Nagata S, Golstein P. (1995) The Fas death factor. Science 267: 1449-1456.

2. Bellgrau D, Duke RC. (1999) Apoptosis and CD95 ligand in immune privileged sites. Int. Rev. Immunol. 18: 547-562.

3. Green DR, Ware CF. (1997) Fas-ligand: privilege and peril. Proc. Natl. Acad. Sci. U.S.A. 94: 5986-5990.

4. Zhang H, Yang Y, Horton JL, et al. (1997) Amelioration of collagen-induced arthritis by CD95 (Apo-1/Fas)-ligand gene transfer. J. Clin. Invest. 100: 1951-1957.

5. Lau H, Yu M, Fontana A, Stoeckert C. (1996) Prevention of islet allograft rejection with engineered myoblasts expressing FasL in mice. Science 273: 109-111.

6. Li XK, Okuyama T, Tamura A, et al. (1998) Prolonged survival of rat liver allografts transfected with Fas ligandexpressing plasmid. Transplantation 66: 1416-1423.

7. Swenson KM, Ke B, Wang T, et al. (1998) Fas ligand gene transfer to renal allografts in rats: effects on allograft survival. Transplantation 65: 155-160.

8. Min WP, Gorczynski R, Huang XY, et al. (2000) Dendritic cells genetically engineered to express Fas ligand induce donor-specific hyporesponsiveness and prolong allograft survival. J. Immunol. 164: 161-167.

9. Seino K, Kayagaki N, Tsukada N, Fukao K, Yagita H, Okumura K. (1997) Transplantation of CD95 ligand-expressing grafts: influence of transplantation site and difficulty in protecting allo- and xenografts. Transplantation 64: 1050-1054.

10. Allison J, Georgiou H, Strasser A, Vaux D. (1997) Transgenic expression of CD95 ligand on islet beta cells induces a granulocytic infiltration but does not confer immune privilege upon islets allografts. Proc. Natl. Acad. Sci. U.S.A. 94: 3943-3947.

11. Takeuchi T, Ueki T, Nishimatsu H, et al. (1999) Accelerated rejection of Fas ligand-expressing heart grafts. J. Immunol. 162: 518-522.

12. Kiener PA, Davis PM, Rankin BM, et al. (1997) Human monocytic cells contain high levels of intracellular Fas ligand: rapid release following cellular activation. J. Immunol. 159: 1594-1598. 
13. Bossi G, Griffiths GM. (1999) Degranulation plays an essential part in regulating cell surface expression of Fas ligand in T cells and natural killer cells. Nat. Med. 5: 90-96.

14. Haimovitz-Friedman A, Cordon-Cardo C, Bayoumy S, et al. (1997) Lipopolysaccharide induces disseminated endothelial apoptosis requiring ceramide generation. J. Exp. Med. 186: $1831-1841$.

15. Dong C, Wilson JE, Winters GL, McManus BM. (1996) Human transplant coronary artery disease: pathological evidence for Fas-mediated apoptotic cytotoxicity in allograft arteriopathy. Lab. Invest. 74: 921-931.

16. Laurence J, Mitra D, Steiner M, Staiano-Coico L, Jaffe E. (1996) Plasma from patients with idiopathic and human immunodeficiency virus-associated thrombotic thrombocytopenic purpura induces apoptosis in microvascular endothelial cells. Blood 87: 3245-3254.

17. Rafi AQ, Zeytun A, Bradley MJ, et al. (1998) Evidence for the involvement of Fas ligand and perforin in the induction of vascular leak syndrome. J. Immunol. 161: 3077-3086.

18. Han DK, Haudenschild CC, Hong MK, Tinkle BT, Leon MB, Liau G. (1995) Evidence for apoptosis in human atherogenesis and in a rat vascular injury model. Am. J. Pathol. 147: 267-277.

19. Janin A, Deschaumes C, Daneshpouy M, et al. (2002) CD95 engagement induces disseminated endothelial-cell apoptosis in vivo: immunopathological implications. Blood 99: 294-297.

20. Sata M, Walsh K. (1998a) TNFalpha regulation of Fas ligand expression on the vascular endothelium modulates leukocyte extravasation. Nat. Med. 4: 415-420.

21. Cardier JE, Schulte T, Kammer H, Kwak J, Cardier M. (1999) Fas (CD95, APO-1) antigen expression and function in murine liver endothelial cells: implications for the regulation of apoptosis in liver endothelial cells. FASEB J. 13: 1950-1960.

22. Richardson B, Lalwani N, Johnson K, Marks R. (1994) Fas ligation triggers apoptosis in macrophages but not endothelial cells. Eur. J. Immunol. 24: 2640-2645.

23. Sata M, Walsh K. (1998b) Oxidized LDL activates fas-mediated endothelial cell apoptosis. J. Clin. Invest. 102: 1682-1689.

24. Aoudjit F, Vuori K. (2001) Matrix attachment regulates Fasinduced apoptosis in endothelial cells: a role for c-Flip and implications for anoikis. J. Cell Biol. 152: 633-643.

25. Kaplan H, Leibole M, Tezel T, Ferguson T. (1999) Fas ligand (CD95 ligand) controls angiogenesis beneath the retina. Nat. Med. 5: 292-297.

26. Sata M, Walsh K. (1998c) Endothelial cell apoptosis induced by oxidized LDL is associated with the downregulation of the cellular caspase inhibitor FLIP. J. Biol. Chem. 273: 3310333106.

27. Suhara T, Mano T, Oliveira B, Walsh K. (2001) Phosphatidylinositol 3-kinase/Akt signaling controls endothelial cell sensitivity to Fas-mediated apoptosis via regulation of FLICE-inhibitory protein (FLIP). Circ. Res. 89: 13-19.

28. Tran H, Grey S, Anrather J, Steinhâuslin F, Bach F, Winkler H. (1998) Regulated and endothelial cell-specific expression of Fas ligand. Transplantation 66: 1126-1131.

29. Charreau B, Tesson L, Soulillou JP, Pourcel C, Anegon I. (1996) Transgenic rats: technical aspects and models. Transgenic. Res. 5: 223-234.
30. Charreau B, Menoret S, Tesson L, et al. (1999) Protection against hyperacute xenograft rejection of transgenic rat hearts expressing human decay accelerating factor (DAF) transplanted into primates. Mol. Med. 5: 617-630.

31. Onel K, Ashani D, Nicolic-Zugic J, Lavy E, Elkon K. (1995) Expression and function of the murine CD95/Fas/APO-1 receptor in relation to B cell ontogeny. Eur. J. Immunol. 25: 940- 945.

32. Kayagaki N, Yamaguchi N, Nagao F, et al. (1997) Polymorphism of murine Fas ligand that affects the biological activity. Proc. Natl. Acad. Sci. U.S.A. 94: 3914-3919.

33. Brouard S, Otterbein LE, Anrather J, et al. (2000) Carbon monoxide generated by heme oxygenase 1 suppresses endothelial cell apoptosis. J. Exp. Med. 192: 1015-1026.

34. David A, Coupel-Clauce H, Chetritt J, et al. (1998) Antiadenovirus immune responses in rats are enhanced by interleukin- 4 but not interleukin- 10 produced by recombinant adenovirus. Hum. Gene Ther. 9: 1755-1768.

35. Stratford-Perricaudet LD, Makeh I, Perricaudet M, Briand P. (1992) Widespread long-term gene transfer to mouse skeletal muscles and heart. J. Clin. Invest. 90: 626-630.

36. Wrighton CJ, Hofer-Warbinek R, Moll T, Eytner R, Bach FH, de Martin R. (1996) Inhibition of endothelial cell activation by adenovirus-mediated expression of I kappa B alpha, an inhibitor of the transcription factor NF-kappa B. J. Exp. Med. 183: 1013-1022.

37. Merrick A, Shewring L, Sawyer G, Gustafsson K, Fabre JW. (1996) Comparison of adenovirus gene transfer to vascular endothelial cells in cell culture, organ culture, and in vivo. Transplantation 62: 1085-1089.

38. Tesson L, Ménoret S, Charreau B, Soulillou J-P, Anegon I. (1999) Endothelial expression of Fas ligand in transgenic rats under the temporal control of a tetracycline-inducible system. Transplant. Proc. 31: 1533-1534.

39. Sata M, Suhara T, Walsh K. (2000) Vascular endothelial cells and smooth muscle cells differ in expression of Fas and Fas ligand and in sensitivity to Fas ligand-induced cell death: implications for vascular disease and therapy. Arterioscler. Thromb. Vasc. Biol. 20: 309-316.

40. Takahashi T, Tanaka M, Inazawa J, Abe T, Suda T, Nagata S. (1994) Human Fas ligand: gene structure, chromosomal location and species specificity. Int. Immunol. 6: 1567-1574.

41. Sata M, Luo Z, Walsh K. (2001) Fas ligand overexpression on allograft endothelium inhibits inflammatory cell infiltration and transplant-associated intimal hyperplasia. J. Immunol. 166: 6494-6971.

42. Bannerman D, Tupper J, Ricketts W, Bennett F, Winn R, Harlan J. (2001) A constitutive protective pathway protects endothelial cells from lipopolysaccharide-induced apoptosis. J. Biol. Chem. 276: 14924-14932.

43. Irmler $M$, Thome $M$, Hahne $M$, et al. (1997) Inhibition of death receptor signals by cellular FLIP. Nature 388: 190-195.

44. Cheng J, Liu C, Yang P, Zhou T, Mountz JD. (1997) Increased lymphocyte apoptosis in Fas ligand transgenic mice. J. Immunol. 159: 674-684.

45. Batteux F, Lores P, Bucchini D, Chiocchia G. (2000) Transgenic expression of Fas ligand on thyroid follicular cells prevents autoimmune thyroiditis. J. Immunol. 164: 1681-1688. 